HNO 2022 $\cdot 70: 24-32$

https://doi.org/10.1007/s00106-021-01022-3

Angenommen: 11. Januar 2021

Online publiziert: 6. April 2021

๑) Der/die Autor(en) 2021

J. Schipper' - A. Albrecht ${ }^{1}$ T. Klenzner' $\cdot$ M. Wagenmann ${ }^{1} \cdot$ K. Schaumann ${ }^{1}$.

D. Hänggi ${ }^{2}$. J. F. Cornelius ${ }^{2}$

' Universitätsklinik für Hals-, Nasen- und Ohrenheilkunde und Poliklinik, Heinrich-Heine-Universität Düsseldorf, Düsseldorf, Deutschland

${ }^{2}$ Universitätsklinik für Neurochirurgie, Heinrich-Heine-Universität Düsseldorf, Düsseldorf, Deutschland

\title{
Interdisziplinäre Chirurgie der zentralen Schädelbasis - aktueller Stand
}

Darüber hinaus führen solche Kadaverstudien auch zu einer Verbesserung der Lernkurve, bevor es in den Operationssaal geht. Allerdings ergeben sich bei der klinischen Umsetzung solcher neu zu etablierender Zugangswege, auch bei noch so guter Vorbereitung und Präparation, nicht vorhersehbare Probleme. Insbesondere venöse Sickerblutungen aus den unterschiedlichsten Weichteilstrukturen mit Verlegung der endoskopischen oder mikroskopischen Sicht stellen dabei gelegentlich eine Herausforderung dar. Dies kann dann dazu führen, dass unter Umständen neue Operationskorridore sich in der klinischen Praxis entgegen den Erfahrungen aus den Kadaverstudien doch nicht bewähren und man auf bisher etablierte Operationsverfahren wieder zurückgreift. Auch solche Studienund Erfahrungsergebnisse, auch wenn sie unter Umständen Irrwege darstellen, sind für die Weiterentwicklung der Schädelbasischirurgie von erheblicher Bedeutung.

Die Entwicklung sog. neuer einzeitiger oder mehrzeitiger „Multi-Port-Zugänge“ im Vergleich zu den bisherigen konventionellen „Single-Port-Zugängen“ ermöglichen durch kombinierten Einsatz von Mikroskop und Endoskop mit Videoketten zur Visualisierung des Operationssitus neue perspektivische Aufsichten sowohl auf die Zielpathologie als auch auf die den chirurgischen Manipulationskorridor verlegenden vitalen Strukturen wie Hirnnerven oder die A. carotis interna. So kann man etwa neben dem Manipulationskorridor durch
Präparation eines oder mehrerer zusätzlicher minimal-invasiver Gewebstunnel mit Einführen einer 30 - oder $45^{\circ}$-Optik die Zielpathologie oder andere bedeutsame Strukturen erstmalig zusätzlich zum jeweiligen Manipulationskorridor auch von der Seite oder von hinten betrachten [28]. Dieser perspektivische „Kubismus“ der jeweiligen Zielstruktur ermöglicht eine noch sicherere chirurgische Exploration im Bereich der zentralen Schädelbasis. Der obligatorische Einsatz der Navigation dient dabei nur der Plausibilisierung des jeweiligen Operationsschritts. Geführt wird der Operateur dabei ausschließlich durch die Visualisierung des jeweiligen Operationssitus und der so sichtbar werdenden Anatomie mit ihren Leitstrukturen. Die Hersteller von Mikroskopen oder anderer Visualisierungssysteme haben sich bereits auf diese neuen Operationstechniken eingestellt und bieten beispielsweise Mikroskope in Kombination mit flexiblen Endoskopen oder sog. stationären Exoskopen in Kombination mit Endoskopen in 4K-Technologie an. Sämtliche verfügbaren perspektivischen Bilddaten inkl. Messdaten vom Neuromonitoring oder von der Navigation werden dann in einer für den Operateur optimalen ergonomischen Position auf einen einzigen Masterbildschirm projiziert [33].

Ein nächster Schritt in die Zukunft wird bei mehrzeitigen Multi-Port-Zugängen die "geodetected navigated visualisation" sein, das bedeutet, dass korrespondierend $\mathrm{zu}$ dem navigierten 




Abb. $1<$ Operative Zugangswege zur zentralen Schädelbasis. „Multi-Port-Zugänge": $A$ transnasal-transsphenoidal (endoskopisch), $B$ subfrontal, subtemporal, transzygomatisch, transpterygonal, $C$ transpetrös, translabyrinthär, $D$ anterosigmoidal, E retrosigmoidal und $F$ subokzipital (von schräg unten kommend)
Zielpunkt das entsprechende intraoperative Bild oder die Videosequenz aus der Voroperation neben dem aktuellen intraoperativen Situs eingeblendet wird. Durch den Vergleich mit den historischen Bilddaten aus einer möglichen anderen Perspektive erhält der Operateur wertvolle Informationen für das weitere mehrzeitige Vorgehen. Allerdings verlangt eine solche Technologie erhebliche Speicherkapazitäten sowie sehr schnelle Grafikkarten, die derzeit noch nicht flächendeckend vorhanden sind. Durch eine detaillierte Auflösung des „fibertracking“ wird es in der Zukunft präoperativ wertvolle Hinweise über den Verlauf der neuronalen Bahnen für die Op.-Planung geben. Der naturgemäß deutlich verlängerte Zugangsweg zur zentralen Schädelbasis, ob nun ausgehend von der Fronto- oder Laterobasis oder vom Splanchnokranium kommend, engt den chirurgischen Manipulationskorridor deutlich ein, wenn man nicht andere den Zugangsweg kreuzende Vitalstrukturen unnötig invalidisieren will. Die Präparation an der Zielpathologie ist damit durch den Eingangsdurchmesser des Manipulationskorridors und der sich daraus ergebenden verkürzten Hebelwege eingeschränkt. „Powered instruments“ wie der CUSA („cavitron ultrasonic surgical aspirator") oder Shaver sind für den notwendigen Gewebsabraum der Zielpathologie sehr hilfreich und schaffen dadurch gleichzeitig Platz für die weitere chirurgische Manipulation und Visualisierung [1]. Sie eignen sich jedoch je nach Konsistenz und Vaskularisierungsgrad nicht für jede Zielpathologie. Bei stark vaskularisierten Zielpathologien im Bereich der zentralen Schädelbasis reduziert eine präoperative endovaskuläre Embolisation das „red-out“ und ermöglicht eine operative Entfernung.

Allen neu entwickelten Operationsverfahren gemeinsam ist die Notwendigkeit einer engen interdisziplinären Zusammenarbeit zwischen dem Chirurgen aus dem Fachgebiet der Hals-NasenOhren-Heilkunde sowie dem Neurochirurgen, da die Pathologien im Bereich der zentralen Schädelbasis nicht haltmachen vor den chirurgischen Grenzen der deutschen Facharztweiterbildung. Die Art und Ausdehnung des notwendigen Operationskorridors werden dabei im Wesentlichen durch die präoperativ radiologisch vermutete Entität der Pathologie sowie durch deren topographische Ausdehnung in der zentralen Schädelbasis bestimmt. Wir haben da- her aufgrund unserer eigenen klinischchirurgischen Erfahrungen der letzten 14 Jahre die jetzt etablierten Zugangsverfahren zur zentralen Schädelbasis unter Berücksichtigung der Entität systematisiert und erörtert auf Grundlage der aktuell publizierten Studienlage.

\section{Patienten und Methoden}

Zur Systematisierung und Würdigung der verschiedenen zu erörternden operativen Zugangswege zur zentralen Schädelbasis wurden retrospektiv, ausschließlich qualitativ und deskriptiv die Operationsberichte einzelner Patienten ausgewertet, die laut Beschluss der hiesigen interdisziplinären Schädelbasiskonferenz gemeinsam mit der Neurochirurgie und der Hals-Nasen-Ohren-Heilkunde/ Kopf- und Halschirurgie in der Zeit zwischen 2006 und 2019 mit Pathologien an der zentralen Schädelbasis chirurgisch behandelt wurden. Für eine Systematisierung wurden die Kriterien chirurgischer Zugangsweg, komplette oder inkomplette Sanierung der Pathologie, Art des multimodalen Therapiekonzepts (Chirurgie als alleinige, neoadjuvante oder adjuvante Therapiemaßnahme) sowie Erst- oder Rezidivtherapie erfasst. Chirurgisches Ziel jeder malignen Pathologie im Bereich der zentralen Schädelbasis war die komplette Entfernung oder aber auch die Diagnosesicherung bei systemischen malignen Erkrankungen. Lediglich bei den benignen Pathologien einschließlich entzündlicher Prozesse war zum Funktionserhalt endokranieller neurovaskulärer Strukturen eine inkomplette bzw. subtotale Resektion unter Umständen angestrebt.

\section{Darstellung und Diskussion der Ergebnisse}

Die chirurgischen Zugangswege zur zentralen Schädelbasis ließen sich nachfolgend kategorisieren, teilweise auch in Kombination derselben, als Multi-PortZugänge: transnasal-transsphenoidal, subfrontal, subtemporal, transzygomatisch, transpterygonal, transpetrös, translabyrinthär und subokzipital (• Abb. 1). Maßgebend für die Wahl des Zugangswegs waren die Lokalisation und Art 
HNO 2022 · 70:24-32 https://doi.org/10.1007/s00106-021-01022-3

(c) Der/die Autor(en) 2021

\section{J. Schipper · A. Albrecht · T. Klenzner · M. Wagenmann · K. Schaumann · D. Hänggi · J. F. Cornelius Interdisziplinäre Chirurgie der zentralen Schädelbasis - aktueller Stand}

\section{Zusammenfassung}

Hintergrund. Die Schädelbasis stellt eine chirurgisch hochkomplexe Einheit dar und ist häufig nur über kombinierte Zugangswege erreichbar. Neu entwickelte Operationstechniken mit Verwendung von mikrochirurgischen Visualisierungsverfahren und aktiven Instrumenten („powered instruments") sowie "Multi-Port-Zugänge" ermöglichen neue, weniger traumatische Operationskorridore. Hierfür ist eine enge interdisziplinäre Zusammenarbeit zwischen dem Chirurgen aus dem Fachgebiet der Hals-Nasen-Ohren-Heilkunde sowie dem Neurochirurgen notwendig. Die aktuell etablierten Zugangsverfahren zur zentralen Schädelbasis werden aufgrund eigener klinischer Erfahrungen und unter Berücksichtigung der Entität systematisiert und in Bezug auf die aktuelle Studienlage erörtert.
Material und Methode. Es erfolgte eine retrospektive, qualitative und deskriptive Auswertung der Operationsberichte einzelner Patienten, die in der Zeit zwischen 2006 und 2019 mit Pathologien an der zentralen Schädelbasis chirurgisch gemeinsam von der Neurochirurgie und der Hals-Nasen-OhrenHeilkunde/Kopf- und Halschirurgie behandelt wurden.

Ergebnisse. Die chirurgischen Zugangswege zur zentralen Schädelbasis ließen sich nachfolgend kategorisieren, teilweise auch in Kombination derselben, als sog. MultiPort-Zugänge: transnasal-transsphenoidal, subfrontal, subtemporal, transzygomatisch, transpterygonal, transpetrös, translabyrinthär und subokzipital. Maßgebend für die Wahl des Zugangswegs waren die Lokalisation und Art der Pathologie, sowie der mögliche Anspruch auf Funktionserhalt und Komplettentfernung. Schlussfolgerung. Aufgrund der Komplexität der Strukturen der zentralen Schädelbasis, der unterschiedlichsten Tumorentitäten und der benötigten Fachkompetenz unterschiedlicher Facharztdisziplinen bleibt die Chirurgie der zentralen Schädelbasis eine Herausforderung, der man sich nur an speziellen, nach den Kriterien der Gesellschaft für Schädelbasischirurgie e.V. zertifizierten Kompetenzzentren stellen sollte.

Schlüsselwörter

Multi-Port-Zugang · Pathologien der zentralen Schädelbasis · Aktive Instumente · Minimal invasive Chirurgie - Mikrochirurgische Visualisierungsverfahren

\section{Interdisciplinary surgery to the central skull base-current status}

\section{Abstract}

Background. The skull base is a surgically complex unit and is often only accessible via combined access routes. Newly developed surgical techniques using microsurgical visualization procedures and active instruments ("powered instruments") as well as multiport accesses enable new, less traumatic surgical corridors. This requires close interdisciplinary cooperation between ENT and neurosurgeons. Currently established access routes to the central skull base are systematized based on the authors' own clinical experience, and discussed in relation to the entity and the current study situation.

Materials and methods. A retrospective, qualitative, and descriptive evaluation of the surgical reports of patients with pathologies of the central skull base who were jointly treated by neurosurgery and otorhinolaryngologic/head and neck surgery between 2006 and 2019 was performed. Results. The surgical access routes to the central skull base can be categorized as so-called multiport access routes, partly also in combination, as follows: transnasaltranssphenoidal, subfrontal, subtemporal, transzygomatic, transpterygonal, transpetrous, translabyrinthine, and suboccipital. The choice of access route was based on the location and type of pathology, its inflammatory or space-occupying (benign or malignant tumor) nature, and the possibilities of functional preservation and complete removal.

Conclusion. Due to the complexity of central skull base structures, the different tumor entities, and the required expertise of different medical specialties, surgery of the central skull base remains a challenge and should only be performed at special competence centers certified according to the criteria of the German Society of Skull Base Surgery.

\section{Keywords}

Multiport access routes - Pathologies of the central skull base - Powered instruments . Minimally invasive surgery $\cdot$ Microsurgical visualization procedures der Pathologie, ob entzündlich oder raumfordernd (benigner oder maligner Tumor) sowie der mögliche Anspruch auf Funktionserhalt und Komplettentfernung (•Tab. 1). Maligne Pathologien der zentralen Schädelbasis wachsen im Gegensatz zu den benignen Pathologien lokal destruierend und können je nach Entität auch metastasieren. Häufigste maligne Pathologien im Bereich der zentralen Schädelbasis sind Karzinome und Sarkome. Bei den Karzinomen finden sich in erster Linie Plattenepithel- karzinome und Adenokarzinome, seltener adenoidzystische Karzinome oder Metastasen, sowie nachfolgend Meningeome (WHO-Grad III, World Health Organization). Bei den Sarkomen sind an erster Stelle die Chondrosarkome zu nennen.

Transnasale bzw. transnasal-transsphenoidale Zugänge wurden bei benignen und malignen Raumforderungen eingesetzt, welche lokal auf den Clivus sowie den kraniozervikalen Übergang begrenzt waren und jeweils medial der
A. carotis interna (ACI) lagen. Typische benigne Tumoren sind Hypophysenadenome, frontobasale Meningeome sowie Kraniopharyngeome. $\mathrm{Zu}$ den malignen Tumoren der Region zählen u. a. lokal aggressive Chordome, Ästhesioneuroblastome, Plattenepithel- und Adenokarzinome. Mit Infiltration der Dura, aber ohne wesentliche Ausdehnung nach intrazerebral, werden sie üblicherweise transnasal reseziert. Die eingeschränkte Möglichkeit der chirurgischen Manipulation und Kontrolle, insbesondere 
Tab. 1 Übersicht über die verschiedenen histopathologischen und topographischen Entitäten, den chirurgischen Zugangsweg, das intraoperative

Visualisierungssystem (endoskopisch $E$, mikroskopisch $M$ ) sowie die Therapiestrategie ggf. mit ergänzenden adjuvanten Therapieverfahren. Ähnlich

wie beim Plattenepithelkarzinom kann auch bei anderen Entitäten über den Einsatz von Biologika nachgedacht werden, hierfür fehlen allerdings aktuell

studienkontrollierte Daten.Die Entscheidung über das individuelle, ggf. multimodale Therapiekonzept sollte immer im Rahmen einer interdisziplinären

Schädelbasiskonferenz getroffen werden. In der vorliegenden Systematik wurden Fehlbildungen nicht berücksichtigt

Entität

Chirurgischer Zugangsweg und Visualisie- Therapiestrategie

Adjuvante Therapie

rungssystem (endoskopisch: $E$, mikrosko-

pisch: M)

\begin{tabular}{|c|c|c|c|}
\hline \multicolumn{4}{|l|}{ Histopathologische Entität } \\
\hline Hypophysenadenome & Transnasal-transsphenoidal $E / M$ & Komplette Resektion ${ }^{\text {b }}$ & $\begin{array}{l}\text { Bei Residuum/Rezidiv: (stereotak- } \\
\text { tische) Strahlentherapie (SRS/SRT) }\end{array}$ \\
\hline \multirow[t]{2}{*}{$\begin{array}{l}\text { Frontobasale Meningeo- } \\
\text { me }\end{array}$} & $\begin{array}{l}\text { Transkraniell (z. B. „Trans-Eyebrow-Zugang”, } \\
\text { Minikraniotomien) } M \text {, ggf. E assistiert }\end{array}$ & \multirow[t]{2}{*}{ Komplette Resektion ${ }^{\mathrm{b}}$} & \multirow{2}{*}{$\begin{array}{l}\text { Bei Residuum/Rezidiv od. höhe- } \\
\text { rem WHO-Grad: (stereotaktische) } \\
\text { Strahlentherapie (SRS/SRT) }\end{array}$} \\
\hline & Transnasal-transplanum $E$ & & \\
\hline \multicolumn{4}{|l|}{ Chordome } \\
\hline $\begin{array}{l}\text { Median gelegene, kleine } \\
\text { bis mittelgroße Tumoren }\end{array}$ & $\begin{array}{l}\text { Transnasal, transclival, ggf. ergänzt durch } \\
\text { transoral } E\end{array}$ & \multirow[t]{4}{*}{ Radikalchirurgische Resektion ${ }^{\text {a }}$} & \multirow[t]{4}{*}{$\begin{array}{l}\text { Hochenergetische Partikelbestrah- } \\
\text { lung }\end{array}$} \\
\hline \multirow[t]{3}{*}{ Große Tumoren } & $\begin{array}{l}\text { Multi-Port-Zugang: transnasal, transsphenoi- } \\
\text { dal, transfazial, transclival } E\end{array}$ & & \\
\hline & $\begin{array}{l}\text { Transzervikal, transkondylär (z. B. „Far-Lateral- } \\
\text { Zugang“) M ggf. mehrzeitiges Vorgehen }\end{array}$ & & \\
\hline & Häufig mit kraniozervikaler Stabilisierung & & \\
\hline $\begin{array}{l}\text { Extrakranielle Neuri- } \\
\text { nome/Paragangliome }\end{array}$ & Transzervikal $M$ & Komplette Resektion ${ }^{\mathrm{b}}$ & $\begin{array}{l}\text { Bei Residuum/Rezidiv: (stereotak- } \\
\text { tische) Strahlentherapie (SRS/SRT) }\end{array}$ \\
\hline $\begin{array}{l}\text { Entzündliche Verände- } \\
\text { rungen }\end{array}$ & $\begin{array}{l}\text { Transmastoidal } M, \text { ggf. ergänzt durch trans- } \\
\text { nasal } E\end{array}$ & $\begin{array}{l}\text { Abstrichentnahme, chirurgische } \mathrm{Ab} \text { - } \\
\text { tragung von Knochensequestern, } \\
\text { Drainage }\end{array}$ & $\begin{array}{l}\text { Langzeitantibiose, ggf. HBO-Thera- } \\
\text { pie }\end{array}$ \\
\hline \multicolumn{4}{|l|}{ Topographische Entität } \\
\hline \multicolumn{4}{|c|}{ Pathologien der Felsenbeinspitze } \\
\hline Dermoide & \multirow[t]{4}{*}{ Subtemporal $M$} & \multirow[t]{2}{*}{ Komplette Resektion ${ }^{\text {b }}$} & - \\
\hline Genuine Cholesteatome & & & - \\
\hline Chondrosarkome & & \multirow[t]{2}{*}{ Radikalchirurgische Resektion ${ }^{\text {a }}$} & $\begin{array}{l}\text { Ggf. hochenergetische Partikelbe- } \\
\text { strahlung }\end{array}$ \\
\hline Metastasen & & & - \\
\hline Cholesteringranulome & Ggf. transnasal-transsphenoidal $E$ & Ggf. Drainage ausreichend & - \\
\hline \multicolumn{4}{|c|}{ Pathologien im Bereich des Sinus cavernosus } \\
\hline Trigeminusneurinome & Subtemporal (extra- oder intradural) $M$ & Komplette Resektion ${ }^{\text {b }}$ & $\begin{array}{l}\text { Bei Residuum/Rezidiv: (stereotak- } \\
\text { tische) Strahlentherapie (SRS/SRT) }\end{array}$ \\
\hline Chondrosarkome & \multirow[t]{4}{*}{$\begin{array}{l}\text { Subtemporal (extra- oder intradural), ggf. } \\
\text { transzygomatisch } M\end{array}$} & Radikalchirurgische Resektion ${ }^{a}$ & $\begin{array}{l}\text { Ggf. hochenergetische Partikelbe- } \\
\text { strahlung }\end{array}$ \\
\hline Riesenzellgranulome & & \multirow[t]{3}{*}{ Komplette Resektion ${ }^{\mathrm{b}}$} & - \\
\hline Osteoblastome & & & - \\
\hline Fibröse Dysplasie & & & Ggf. Bisphoshonate \\
\hline \multicolumn{4}{|c|}{ Tumoren medial der GI. parotis } \\
\hline Pleomorphe Adenome & \multirow[t]{3}{*}{ Transmaxillär $M / E$, transpterygonal, ggf. $E$} & \multirow[t]{3}{*}{ Radikalchirurgische Resektion ${ }^{a}$} & - \\
\hline Adenokarzinome & & & - \\
\hline Plattenepithelkarzinome & & & Ggf. Radio-/Chemotherapie \\
\hline
\end{tabular}



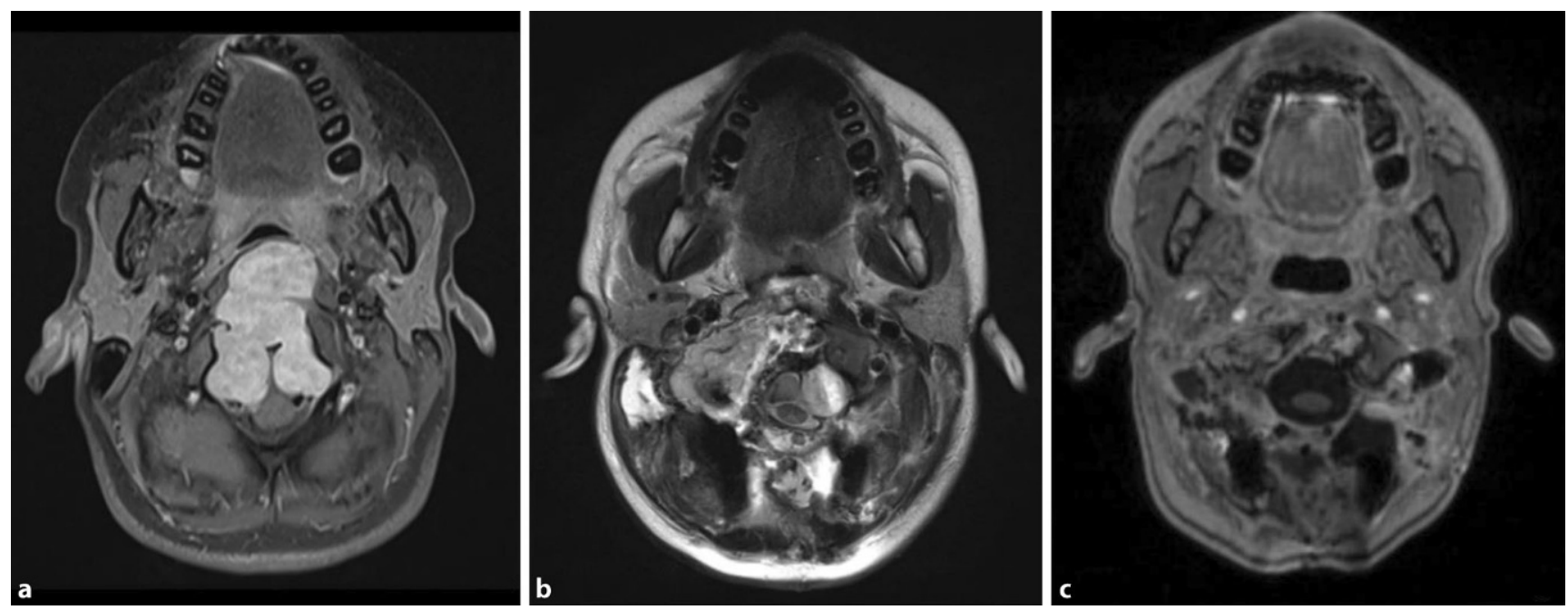

Abb. $2 \triangle$ MRT (axial). a Ausgedehntes Clivuschordom mit Kompression des Hirnstamms und des Rückenmarks sowie beginnender hoher Tetrasymptomatik, $\mathbf{b}$ nach subtotaler Entfernung von rechts über einen extrem lateral transkondylär-transodontoidalen Zugang (ELTO) und Defektauffüllen mit Bauchfett sowie eine okzipitozervikale Stabilisierung, c 6-Monats-Follow-up nach Entfernung des Resttumors von links über einen transkondylären Zugang (ELTO)

bei Blutungen lateral der ACI, wurden bereits ausführlich durch die Publikationen von Kassam u.a. erörtert, [9-11] sodass man bei Raumforderungen mit Ausdehnung über die ACI nach lateral hinaus im Allgemeinen einen transkraniellen Zugangsweg favorisieren würde. Seltene Ausnahmen stellen im Einzelfall nur benigne Tumoren dar.

Hypophysenadenome können als der Prototyp für den transnasalen endoskopischen Zugangsweg angesehen werden $[21,22]$. Dieser kann mono- oder bilateral erfolgen, was v.a. von Größe und Konsistenz des Tumors abhängt. Der Zugang muss so groß wie nötig sein, um den Tumor sicher und komplett entfernen zu können. Andererseits ist aber für den Erhalt einer guten Lebensqualität ein möglichst geringes Zugangstrauma in der Nase wichtig, d.h. Schonung und Erhalt von Nasenschleimhaut, -septum und -muscheln. Die knöcherne bzw. durale Rekonstruktion der Schädelbasis erfolgt in Abhängigkeit von der Defektgröße und dem erwarteten Risiko einer Durafistel [19]. Bei kleineren Defekten mit niedrigem Liquorfluss sind kleinere Abdichtungsmaßnahmen meist ausreichend. Bei größeren Defekten und hohem Liquorfluss, z. B. nach Eröffnung des III. Ventrikels und des Diaphragma sellae, kann durch einen gestielten, vaskularisierten mukoseptalen Lappen n. Hadad im All- gemeinen ein wasserdichter Verschluss gut erreicht werden [3].

Frontobasale Meningeome, uni- oder bilateral der Riechspalte, wurden nach Einführung und stetiger Verbesserung der endoskopischen Techniken zeitweise bevorzugt transnasal operiert. Aufgrund des hierbei erhöhten Risikos einer Hypoder gar Anosmie hat es im Laufe der Zeit dann wieder eine Rückkehr zu den transkraniellen Zugängen gegeben [22]. Die transkraniellen Zugänge haben sich allerdings im Vergleich zu den Anfängen der Schädelbasischirurgie zunehmend $\mathrm{zu}$ weniger invasiven Varianten entwickelt, „Keyhole-Konzept“ [4, 20, 30]. Kleine Hautschnitte, z.B. ein „TransEyebrow-Zugang“ sowie Minikraniotomien führten $\mathrm{zu}$ einer deutlichen Verringerung des Zugangstraumas mit einfacheren und schnelleren postoperativen Heilungsverläufen bei ästhetisch sehr guten Ergebnissen [5, 6]. Die durch Miniaturisierung der Zugänge reduzierte Sicht kann durch ein Endoskop gut kompensiert werden, sog. endoskopischassistierte Mikrochirurgie.

Bei den Chordomen handelt es sich um maligne Knochentumoren mit notochordaler Differenzierung, die sich fast ausschließlich entlang des Achsenskeletts entwickeln. Sie haben ihre Ursprungsmatrix in der phylogenetisch sich bildenden Neuralleiste. Die Neuralleiste wiederum befindet sich in der Medianebene am kra- niozervikalen Übergang. Daher befinden sich Chordome im Bereich der Schädelbasis typischerweise im Clivus, von wo aus sie dann lokal destruierend in alle Richtungen wachsen [13]. Grundsätzlich sollte aufgrund der sehr hohen Rezidivneigung von Chordomen immer eine radikale Tumorentfernung angestrebt werden, auch deswegen, da die Chordome relativ schlecht durch Strahlen- und Chemotherapie allein therapierbar sind [18].

Häufig ist die Stabilität des kraniozervikalen Übergangs bereits durch die Tumordestruktion beeinträchtigt, manchmal wird diese aber auch durch die notwendige radikale Resektion induziert und muss in jedem Fall durch eine kraniozervikale Stabilisierung behandelt werden.

Für median gelegene, kleine bis mittelgroße Chordome ist meist ein transnasal-transclivaler, ggf. ergänzt durch einen transoralen Zugangsweg geeignet. Große Chordome (-Abb. 2 und 3) müssen unter Umständen über einen MultiPort-Zugang reseziert werden, ggf. sogar mehrzeitig, beispielsweise kombiniert transnasal, transzervikal und/oder transkondylär. Die adjuvante Therapie der ersten Wahl ist derzeit die postoperative hochenergetische Partikelbestrahlung durch Protonen oder Karbonionen [17]. Da die Radiatio durch etwaiges Osteosynthesematerial beeinflusst wer- 

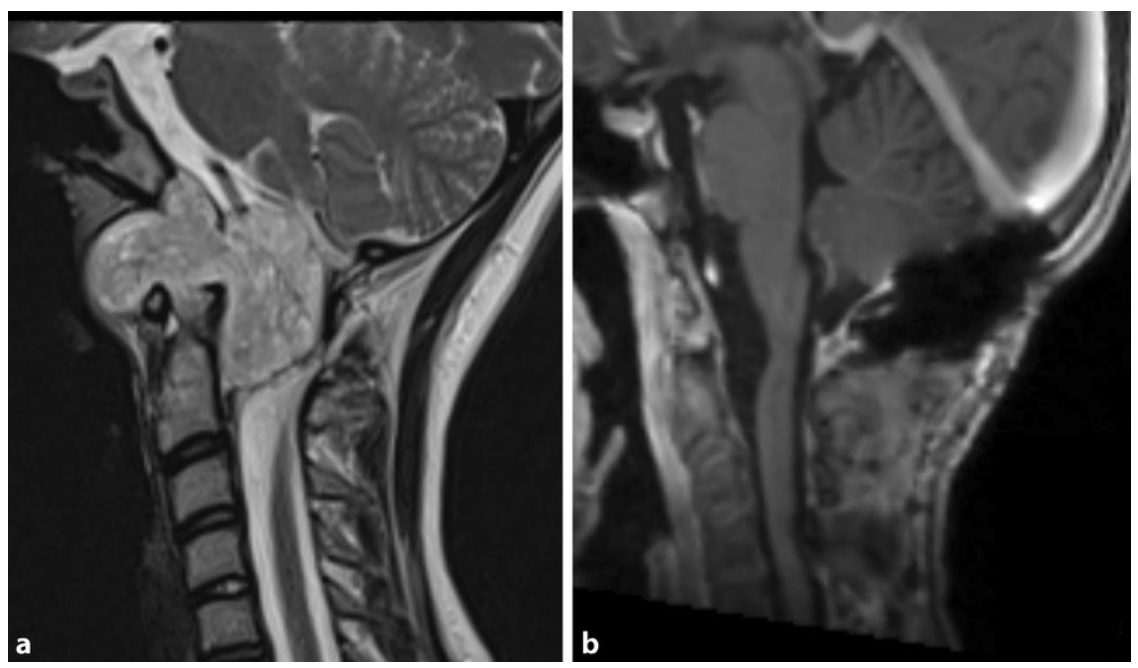

Abb. 3 ॥ MRT (sagittal). a Präoperativ. Großes Clivuschordom mit Extension nach retropharyngeal sowie in die hintere Schädelgrube mit Hirnstammkompression. Zugang: 1. Schritt: transkondylär-transodontoidaler Zugang (von rechts) M und E assistiert mit kraniozervikaler Stabilisierung C0-C42. 2. Schritt: transkondylärer Zugang (von links) M, b 6-Monats-Follow-up nach zweizeitiger Op. und Protonenbestrahlung: kein Hinweis auf Rest oder Rezidiv, beachte: Suszeptibilitätsartefakte des Osteosynthesematerials

den kann, ist für Chordome eine enge interdisziplinäre Zusammenarbeit und eine sorgfältige multimodale Therapieplanung von besonderer Bedeutung [24]. Die Überlebensprognose der Patienten ist bei einer radikalen Therapie bei Erstmanifestation am besten, weshalb die Behandlung der extrem seltenen Chordome Schwerpunktzentren vorbehalten bleiben sollte [14-16].

Pathologien im Bereich der Felsenbeinspitze lassen sich gut von lateral kommend über einen subtemporalen Zugangsweg resezieren. Da bei diesem Zugangsweg nicht der Meatus acusticus internus das Zielgebiet ist, sondern die Felsenbeinspitze, wird die Kraniotomie temporobasal etwas weiter frontal durchgeführt und der weitere Zugang dann extradural unterhalb des Temporallappens [27]. Dieser sollte beim Lagern durch eine leichte Kopfflexion bodenwärts dazu gebracht werden, sich der Schwerkraft folgend vom Boden der mittleren Schädelgrube abzuheben. Dies kann durch eine zusätzliche Hirnrelaxation mit Lumbaldrainage und vorheriger Mannitgabe unterstützt werden. Das Einsetzen eines Spatels sollte möglichst vermieden werden, um den Temporallappen nicht zu schädigen. Weiterhin muss besonders auf das horizontale bzw. petrosale Segment der A. carotis interna Rücksicht genommen werden, welche im Felsenbein nur durch einen dünnen Knochen bzw. bisweilen gar nicht knöchern gedeckt verläuft. Typische Pathologien sind gutartige Tumoren wie Dermoide, genuine Cholesteatome, Cholesteringranulome, aber auch maligne Raumforderungen wie Chondrosarkome oder Metastasen. Bei den Cholesteringranulomen ist $\mathrm{zu}$ bedenken, dass eine sichere vollständige Resektion nicht immer möglich ist und an eine ausreichende Drainage für das nachlaufende Zellsekret aus den verbleibenden Granulomzellen entweder nach transnasal oder transtympanal gedacht auch, je nach anatomischer Situation, eine transnasal-transsphenoidale Exploration der Felsenbeinspitze von Vorteil sein, ggf. auch als Multi-Port-Zugang $[23,25]$. Bei der Wahl des transnasalen Zugangswegs ist v.a. die präoperativ computertomographisch zu bestimmende Größe der lateralen Wand des Sinus sphenoidalis sowie dessen topographische Lage zum Recessus caroticoopticus entscheidend, inwieweit ein solcher $\mathrm{Zu}$ gangsweg überhaupt möglich ist.

Der subtemporale Zugangsweg kann durch einen transzygomatischen Zugang erweitert werden. Diese Kombination ermöglicht es dann gut, zentral gelegenen Pathologien im Bereich des Sinus cawerden muss. In diesen Fällen kann vernosus mit Extension in die Fossa pterygopalatina zu erreichen (GlascockDreieck). In erster Linie erfolgt auch dieser Zugang extradural und kann aber im Zielbereich ggf. durch ein intradurales Vorgehen ergänzt werden. Neben den typischen Trigeminusneurinomen und Chondrosarkomen gibt es andere seltenere Pathologien in diesem Bereich, wie z. B. Riesenzellgranulome, Osteoblastome oder eine fibröse Dysplasie. Die Schlüsselprozedur bei diesem Zugangsweg ist die temporäre Rotation des vorderen Temporalmuskelanteils nach frontokaudal unter Schonung des Ramus frontalis nervi facialis sowie die osteoplastische Transposition des Os zygomaticum [26]. Damit erhält man einen optimalen Überblick auf die Seitenwand des Sinus cavernosus sowie auf die kranialen Strukturen der Fossa pterygopalatina, ohne dass man dadurch die Substanz und Funktion der Pterygoidmuskulatur verletzt. Dieses kann bleibende Funktionsstörungen der Kaumuskulatur nach sich ziehen (- Abb. 4 und 5). Ebenso ist hierdurch auch keine Sichteinschränkung durch den Processus coronoideus gegeben. Tiefer gelegene Pathologien im Bereich der mittleren oder unteren Etage der Fossa pterygopalatina lassen sich dagegen besser transnasal, transpterygonal bzw. transmaxillär endoskopisch angehen. Lediglich nachteilig dabei ist unter Umständen die Traumatisierung und anschließende Vernarbung der Pterygoidmuskulatur mit nachfolgenden Einschränkungen in der Kiefergelenkbewegung. Zudem kommt es durch diffuse Sickerblutungen im Bereich des venösen Plexus pterygoideus häufig zur endoskopischen Sichteinschränkung, die diesen Zugangsweg dann zu einer chirurgischen Geduldsprobe werden lassen. Die A. maxillaris wird in der Regel geclippt. Der radiologisch sichtbare Clip ist dann später im Follow-up eine wichtige Landmarke zur topographischen Orientierung in dem chirurgisch veränderten Bilddatensatz. Der N. vidianus ist intraoperativ häufig die bessere Nervenleitstruktur als die operative Navigation zur Identifizierung der daruntergelegenen A. carotis interna $[11,12]$.

Anders als bei raumfordernden $\mathrm{Tu}$ morentitäten gestaltet sich das Therapie- 

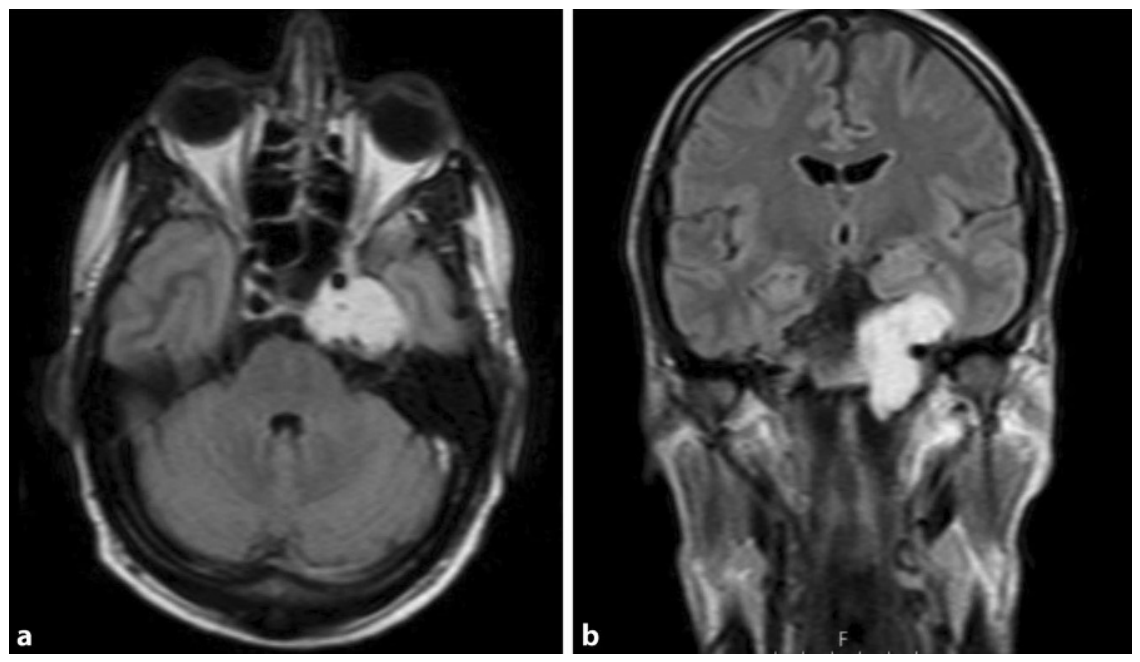

Abb. 4 A Chondrosarkom mit Extension in die Fossa cerebri media, den Sinus cavernosus, die Fossa infratemporalis und nach prävertebral bis HWK1, MRT a axial, b koronar
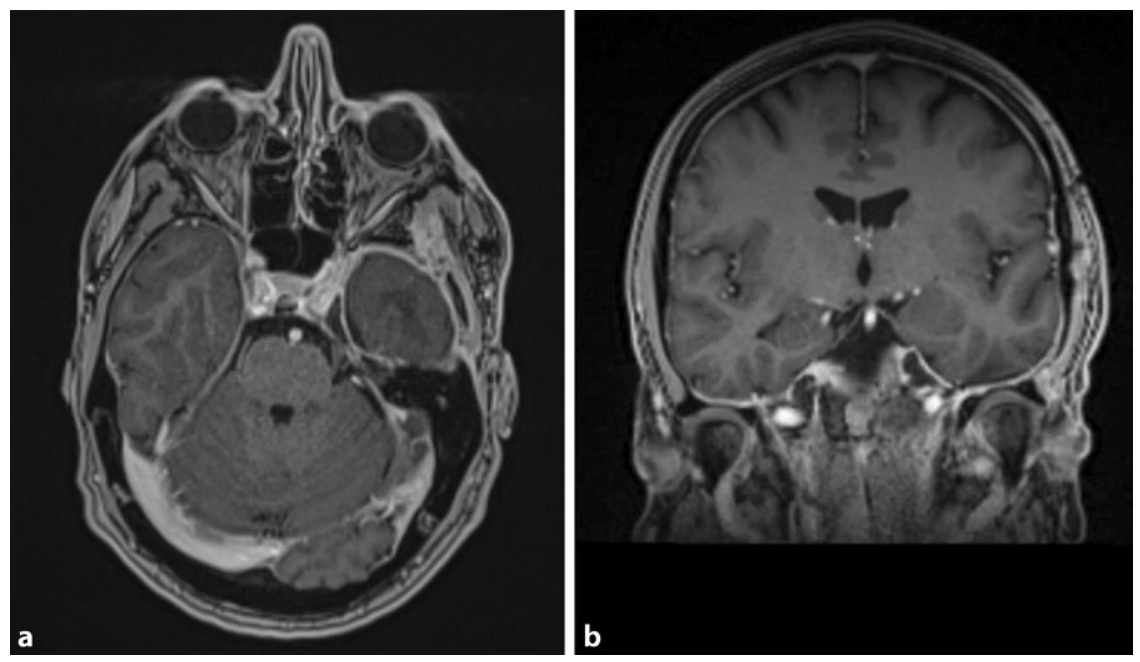

Abb. 5 \& Im 9-monatigen Follow-up bildmorphologisch tumorfrei nach navigierter mikrochirurgischer endoskopisch und dopplersonographisch unterstützter makroskopischer Komplettresektion über einen einzeitigen Multi-Port-Zugang transzygomatisch-subtemporal sowie von hoch zervikal Richtung Schädelbasis, a axial, b koronar

regime bei entzündlichen Veränderungen im Bereich der zentralen Schädelbasis. In der Regel handelt es sich dabei um eine Osteomyelitis oder um eine Ostitis, die radiologisch je nach Entzündungsstadium nicht immer sofort erkennbar sind. Häufig sind dabei klinische Prodromalzeichen wegweisend wie etwa das bekannte „Gradenigo-Syndrom“ oder andere solitäre Hirnnervenausfälle in Kombination mit Kopf- und Gesichtsschmerzen. Ausgangspunkt kann eine Sinusitis oder eine Otitis externa necroticans sein, möglicherweise begünstigt durch eine immunsuppressive Abwehrlage oder bei Diabetes mellitus. Durch die Unspezifität der klinischen Symptomatik sind Patienten oft bereits mit Breitbandantibiotika frustran vorbehandelt, was eine spätere mikrobiologische Charakterisierung zusätzlich erschwert. Darüber hinaus muss man erst einmal durch einen operativen Zugangsweg zur zentralen Schädelbasis die Möglichkeit schaffen, entzündlich abgekapseltes Gewebe zu entfernen und den Bereich sozusagen zu „belüften“. Dies gelingt häufig nur durch ein kombiniert chirurgisches Vorgehen - transmastoidal im Sinne einer Petrosektomie und transnasal über die Keilbeinhöhle und den Nasopharynx. Nur so kann ein repräsentativer Abstrich am Zielort auch von abgekapselten Prozessen aus dem Knochenmaterial gewonnen werden. Ursächlich sind hier häufig Anaerobier oder auch Pseudomonas aeruginosa im Rahmen einer Otitis externa maligna. Neben der notwendigen chirurgischen Abtragung von Knochensequestern und Drainierung zur äußeren Umgebung bei bakteriellen Infekten im Bereich der zentralen Schädelbasis kann v. a. bei Anaerobiern der Einsatz der hyperbaren Sauerstofftherapie den Genesungsprozess deutlich beschleunigen $[7,8]$. In der Regel verlaufen solche Erkrankungen schleichend progredient sowie langwierig und bedürfen daher einer äußerst langen begleitenden Antibiotikatherapie, teilweise über mehrere Monate bis Jahre, einschließlich radiologischer Kontrollen. Zusätzlich fortschreitende Infektionen mit Pilzen im Bereich der zentralen Schädelbasis stellen eine besondere Herausforderung dar und müssen radikalchirurgisch saniert werden, da in solchen Fällen die Mortalität deutlich ansteigt [7].

Medial der Gl. parotis gelegene Tumoren, lateral oder medial eines der beiden Pterygoidflügel wie pleomorphe Adenome aber auch Adeno- oder Plattenepithelkarzinome lassen sich über einen transmaxillär-transpterygonalen Zugangsweg endoskopisch-chirurgisch erfassen. Bei den malignen Tumoren ist im Rahmen multimodaler Therapiekonzepte immer eine radikalchirurgische Sanierung anzustreben. Eine „Piecemeal-Technik" schließt dies aber nicht aus. Chirurgisch-endoskopische Leitstruktur ist dabei der N. infraorbitalis der mit endoskopischer Entfernung des pterygomaxillären Pfeilers in die Fossa pterygopalatina verfolgt wird bis hin zum Ganglion pterygopalatinum. Der anterior des Ganglion pterygopalatinum gelegene Plexus venosus pterygopalatinum verlegt häufig durch venöse Sickerblutungen die endoskopische Sicht und kann den Fortgang der Operation erheblich verzögern. Größere maligne Tumoren im Bereich der Fossa pterygopalatina sollten je nach multimodalem Tumorkonzept ggf. auch extranasal über eine temporäre osteoplastische Maxillektomie mit Exploration der Fossa pterygopalatina radikalchirurgisch entfernt werden. Eine interdisziplinäre Einbindung der Neu- 
rochirurgen für medial der Gl. parotis gelegene Pathologien ist immer dann von Bedeutung, wenn eine Extension nach endokraniell in der präoperativen Bildgebung gesehen oder zumindest stark angenommen wird.

Extrakraniell gelegene Neurinome des $\mathrm{N}$. hypoglossus oder des $\mathrm{N}$. vagus oder vagale Paragangliome, aber auch seltenere ektope Meningeome vergleichbarer Lokalisation können auch alternativ über einen medial des M. digastricus zu präparierenden Zugangsweg angegangen werden. Dabei wird zur Sicherung der A. carotis interna die Fascia cervicalis profunda entlang der Gefäßscheide nach kranial verfolgt bis hin zur Schädelbasis medial des Processus stylomastoideus, um dann den Tumor von kranial nach kaudal zu mobilisieren und zu entfernen. Zur Schonung der kaudalen Hirnnerven sollte in diesem Bereich immer ein entsprechendes intraoperatives Neuromonitoring sichergestellt sein.

\section{Schlussfolgerung}

Maligne Tumoren bedürfen aus onkologischer Sicht immer einer radikalchirurgischen Entfernung, benigne Tumoren können unter Umständen zum Funktionserhalt auch inkomplett reseziert werden. Da die zu behandelnden Pathologien im Bereich der zentralen Schädelbasis häufig eine unmittelbare Nachbarschaft zu vitalen Strukturen aufweisen, müssen zur Wahrung der Lebensqualität im Rahmen multimodaler Tumortherapiekonzepte auf Basis belastbarer Studien in Kombination mit nichtchirurgischen, neoadjuvanten oder adjuvanten Behandlungsverfahren entsprechende Kompromisse entwickelt werden. Diese müssen vor Beginn einer solchen Behandlung in einer durch die Deutsche Gesellschaft für Schädelbasischirurgie (DGSB) zertifizierten, interdisziplinären Schädelbasiskonferenz auf Grundlage der radiologisch-klinischen Diagnostik erörtert und festgelegt werden. Nicht zuletzt wegen möglicher späterer medikolegaler Haftungsansprüche sind diese Entscheidungen immer transparent und nachvollziehbar im Rahmen der notwendigen interdisziplinären
Schädelbasiskonferenzen zu dokumentieren.

In der Regel sind die Entscheidungen der interdisziplinären Schädelbasiskonferenz sehr individuell. Damit ist die Möglichkeit einer Stratifizierung der verschiedenen Tumorentitäten der Schädelbasis für eine Studie statistisch erheblich eingeschränkt. Vor allem ist aufgrund individuell unterschiedlicher Tumorwachstumsmuster letztlich keine sinnvolle Kategorisierung möglich. Nicht zuletzt auch deswegen gibt es für die Malignome im Bereich der zentralen Schädelbasis keine TNM-Klassifikation der WHO. Grundlage jeder therapeutischen Entscheidung ist somit v.a., inwieweit die beteiligten interdisziplinären chirurgischen Fachabteilungen sich in der Lage sehen, die jeweilige Pathologie komplett oder inkomplett chirurgisch zu sanieren. Davon abhängig erfolgt dann die Anpassung einer neoadjuvanten oder adjuvanten Strahlen- oder kombinierten Strahlen-/Chemotherapie bei den malignen Neoplasien [32]. Die chirurgische Radikalität muss darüber hinaus gegen den zu erwartenden, funktionellchirurgischen Flurschaden abgewogen werden.

Aufgrund der Komplexität der Strukturen der zentralen Schädelbasis, der unterschiedlichsten Tumorentitäten und der benötigten Fachkompetenz unterschiedlicher Facharztdisziplinen bleibt die Chirurgie der zentralen Schädelbasis eine Herausforderung, der man sich nur an speziellen zertifizierten Kompetenzzentren stellen sollte.

\section{Korrespondenzadresse}

Prof. Dr. med. Dr. h.c. J. Schipper

Universitätsklinik für Hals-, Nasen- und Ohrenheilkunde und Poliklinik, Heinrich-HeineUniversität Düsseldorf

Moorenstraße 5, 40255 Düsseldorf,

Deutschland

joerg.schipper@med.uni-duesseldorf.de

Funding. Open Access funding enabled and organized by Projekt DEAL.
Hier steht eine Anzeige. Springer 


\section{Einhaltung ethischer Richtlinien}

Interessenkonflikt. J. Schipper, A. Albrecht, T. Klenzner, M. Wagenmann, K. Schaumann, D. Hänggi und J. F. Cornelius geben an, dass kein Interessenkonflikt besteht.

Für diesen Beitrag wurden von den Autoren keine Studien an Menschen oder Tieren durchgeführt. Für die aufgeführten Studien gelten die jeweils dort angegebenen ethischen Richtlinien.

Open Access. Dieser Artikel wird unter der Creative Commons Namensnennung 4.0 International Lizenz veröffentlicht, welche die Nutzung, Vervielfältigung, Bearbeitung, Verbreitung und Wiedergabe in jeglichem Medium und Format erlaubt, sofern Sie den/die ursprünglichen Autor(en) und die Quelle ordnungsgemäß nennen, einen Link zur Creative Commons Lizenz beifügen und angeben, ob Änderungen vorgenommen wurden.

Die in diesem Artikel enthaltenen Bilder und sonstiges Drittmaterial unterliegen ebenfalls der genannten Creative Commons Lizenz, sofern sich aus der Abbildungslegende nichts anderes ergibt. Sofern das betreffende Material nicht unter der genannten Creative Commons Lizenz steht und die betreffende Handlung nicht nach gesetzlichen Vorschriften erlaubt ist, ist für die oben aufgeführten Weiterverwendungen des $\mathrm{Ma}$ terials die Einwilligung des jeweiligen Rechteinhabers einzuholen.

Weitere Details zur Lizenz entnehmen Sie bitte der Lizenzinformation auf http://creativecommons.org/ licenses/by/4.0/deed.de.

\section{Literatur}

1. Tang H, Zhang H, Xie Q et al (2014) Application of CUSA Excel ultrasonic aspiration system in resection of skull base meningeomas. Clin J Cancer Res 26(6):653-657

2. Ciporen JN, Moe KS, Ramanathan D et al (2010) Multiportal endoscopic approaches to the central skull base: a cadaveric study. World Neurosurg 73(6):705-712

3. Hadad G, Bassagasteguy L, Carrau RL et al (2006) A novel reconstructive technique after endoscopic expanded endonasal approaches: vascular pedicle nasoseptal flap. Laryngoscope 116(10):1882-1886

4. Perneczky A (2004) Surgical results, complications and patient satisfaction after supraorbital craniotomy through eyebrow skin incision. In: Proceedings of the Joint Meeting mit der Ungarischen Gesellschaft für Neurochirurgie Deutsche Gesellschaft für Neurochirurgie (DGNC'04) Köln

5. Reisch R, Perneczky A (2005) Ten-year experience with the supraorbital subfrontal approach through an eyebrow skin incision. Neurosurgery $57(4$ Suppl):242-255

6. Reisch R, Perneczky A, Filippi R (2003) Surgical technique of the supraorbital key-hole craniotomy. Surg Neurol 59(3):223-227

7. Mortazavi MM, Khan MA, Quadri SA et al (2018) Cranial osteomyelitis: a comprehensive review of modern therapies. World Neurosurg 111:142-153

8. Sander A, Henze D, Neumann Ketal (2009) Value of hyperbaric oxygen in the treatment of advanced skull base osteomyelitis. Laryngorhinootologie 88(10):641-646
9. Kassam AB, Gardner P, Snyderman C et al (2005) Expanded endonasal approach: fully endoscopic, completely transnasal approach to the middle third of the clivus, petrous bone, middle cranial fossa, and infratemporal fossa. Neurosurg Focus 19(1):E6

10. Kassam A, Carrau RL, Snyderman CH et al (2005) Evolution of reconstructive techniques following endoscopic expanded endonasal approaches. Neurosurg Focus 19(1):E8 (Review.(Dura Verschluß centrale Schädelbasis))

11. Kassam AB, Vescan AD, Carrau RL et al (2008) Expanded endonasal approach: vidian canal as a landmark to the petrous internal carotid artery. J Neurosurg 108(1):177-183

12. Kasemsiri P, Solares CA, Carrau RL et al (2013) Endoscopic endonasal transpterygoid approaches: anatomical landmarks for planning the surgical corridor. Laryngoscope 123(4):811-815

13. McMaster ML, Goldstein AM, Bromley CM et al (2001) Chordoma: incidence and survival patterns in the United States, 1973-1995. Cancer Causes Control 12(1):1-11

14. Bergh P, Kindblom LG, Gunterberg B et al (2000) Prognostic factors in chordoma of the sacrum and mobile spine: a study of 39 patients. Cancer 88(9):2122-2134

15. Schwab JH, Boland PJ, Agaram NP et al (2009) Chordoma and chondrosarcoma gene profile: implications for immunotherapy. Cancer Immunol Immunother 58(3):339-349

16. Jahangiri A, Jian B, Miller $L$ et al (2013) Skull base chordomas: clinical features, prognostic factors, and therapeutics. Neurosurg Clin N Am 24(1):79-88

17. Lu VM, O'Connor KP, Mahajan A et al (2020) Carbon ion radiotherapy for skull base chordomas and chondrosarcomas: a systematic review and metaanalysis of local control, survival, and toxicity outcomes. J Neurooncol 147(3):503-513

18. Boriani S, Chevalley F, Weinstein JN et al (1996) Chordoma of the spine above the sacrum. Treatment and outcome in 21 cases. Spine 21(13):1569-1577

19. Molitch ME (2017) Diagnosis and treatment of pituitary adenomas: a review. JAMA 317(5):516-524

20. Ottenhausen M, Rumalla K, Alalade AF et al (2018) Decision-making algorithm for minimally invasive approaches to anterior skull base meningiomas. Neurosurg Focus 44(4):E7

21. Jho HD, Carrau RL (1997) Endoscopic endonasal transsphenoidal surgery: [] experience with 50 patients. J Neurosurg 87(1):44-51

22. McLaughlin N, Eisenberg AA, Cohan P et al (2013) Value of endoscopy for maximizing tumor removal in endonasal trans-sphenoidal pituitary adenoma surgery. J Neurosurg 118(3):613-620

23. Bloch O, Parsa AT (2013) Skull base chondrosarcoma: evidence-based treatment paradigms. Neurosurg Clin N Am 24(1):89-96

24. Mohyeldin A, Prevedello DM, Jamshid AO et al (2014) Nuances in the treatment of malignant tumors of the clival and petroclival region. Int Arch Otolaryngol 18(Suppl 2):S157-S172

25. Muto J, Prevedello DM, Ditzel Filho LF et al (2016) Comparative analysis of the anterior transpetrosal approach with the endoscopic endonasal approach to the petroclival region. JNeurosurg 125(5):1171-1186

26. Shigeno T, Tanaka J, Atsuchi M (1999) Orbitozygomatic approach by transposition of temporalis muscle and one-piece osteotomy. Surg Neurol 52(1):81-83
27. Zhang J, Hua W, Zhang X et al (2019) Pure endoscopic surgery via subtemporal extradural keyhole approach for middle cranial fossa tumors. World Neurosurg 130:e487-e497

28. Stenin I, Hansen S, Becker Met al (2014) Minimally invasive multiport surgery of the lateral skull base. Biomed Res 2014:379295

29. Ozcan T, Yilmazlar S, Aker S et al (2010) Surgical limits in transnasal approach to opticocarotid region sphenoidale: an anatomic cadaveric study. World Neurosurg 73(4):326-333

30. Fatemi N, Dusik JR, de Paiva Neto MA et al (2009) Endonasal versus supraorbital keyhole removal of craniopharyngeomas and tuberculum sellae meningeomas. Neurosurgery 64(5 Suppl 2):269-284 (discussion 284-86)

31. Peraio S, Chumas P, Nix P et al (2018) From above or from below? That is the question. Comparison of the supraorbital approach with the endonasal approach. A cadaveric study. Br J Neurosurg 35(5):548-552

32. Turri-Zanoni M, Maragliano R, Battaglia $P$ et al (2017) The clinicopathological spectrum of olfactory neuroblastoma and sinonasal neuroendocrine neoplasms: Refinements in diagnostic criteria and impact of multimodal treatments on survival. Oral Oncol 74:21-29. https://doi.org/10. 1016/j.oraloncology.2017.09.010.

33. Ricciardi L, Mattogno PP, Olivi A et al (2019) Exoscope Era: Next Technical and Educational Step in Microneurosurgery. World Neurosurg 128:371-373 